\title{
Optimal (and Benchmark-Optimal) Competition Complexity for Additive Buyers over Independent Items
}

\author{
Hedyeh Beyhaghi \\ Cornell University \\ Ithaca, NY, USA \\ hedyeh@cs.cornell.edu
}

\author{
S. Matthew Weinberg* \\ Princeton University \\ Princeton, NJ, USA \\ smweinberg@princeton.edu
}

\begin{abstract}
The Competition Complexity of an auction setting refers to the number of additional bidders necessary in order for the (deterministic, prior-independent, dominant strategy truthful) Vickrey-ClarkeGroves mechanism to achieve greater revenue than the (randomized, prior-dependent, Bayesian-truthful) optimal mechanism without the additional bidders.

We prove that the competition complexity of $n$ bidders with additive valuations over $m$ independent items is at most $n(\ln (1+$ $m / n)+2$ ), and also at most $9 \sqrt{n m}$. When $n \leq m$, the first bound is optimal up to constant factors, even when the items are i.i.d. and regular. When $n \geq m$, the second bound is optimal for the benchmark introduced by Eden et al.up to constant factors, even when the items are i.i.d. and regular. We further show that, while the Eden et al. benchmark is not necessarily tight in the $n \geq m$ regime, the competition complexity of $n$ bidders with additive valuations over even 2 i.i.d. regular items is indeed $\omega(1)$.

Our main technical contribution is a reduction from analyzing the Eden et al. benchmark to proving stochastic dominance of certain random variables.
\end{abstract}

\section{CCS CONCEPTS}

- Theory of computation $\rightarrow$ Sample complexity and generalization bounds; Algorithmic game theory and mechanism design; Algorithmic mechanism design.

\section{KEYWORDS}

auctions, duality, stochastic dominance, multi-dimensional mechanism design

\section{ACM Reference Format:}

Hedyeh Beyhaghi and S. Matthew Weinberg. 2019. Optimal (and BenchmarkOptimal) Competition Complexity for Additive Buyers over Independent Items. In Proceedings of the 51st Annual ACM SIGACT Symposium on the Theory of Computing (STOC '19), June 23-26, 2019, Phoenix, AZ, USA. ACM, New York, NY, USA, 11 pages. https://doi.org/10.1145/3313276.3316405

\footnotetext{
* Supported by NSF CCF-1717899.

Permission to make digital or hard copies of all or part of this work for personal or classroom use is granted without fee provided that copies are not made or distributed for profit or commercial advantage and that copies bear this notice and the full citation on the first page. Copyrights for components of this work owned by others than the author(s) must be honored. Abstracting with credit is permitted. To copy otherwise, or republish, to post on servers or to redistribute to lists, requires prior specific permission and/or a fee. Request permissions from permissions@acm.org.

STOC '19, fune 23-26, 2019, Phoenix, AZ, USA

(C) 2019 Copyright held by the owner/author(s). Publication rights licensed to ACM. ACM ISBN 978-1-4503-6705-9/19/06 ..\$15.00

https://doi.org/10.1145/3313276.3316405
}

\section{INTRODUCTION}

In the past decade, the TCS community has made radical progress developing the theory of multi-dimensional mechanism design. In particular, it was previously well-understood the optimal multiitem auctions are prohibitively complex, even with just $m=2$ items, and even subject to fairly restricted instances [BCKW15, HN13, HR15, Tha04, Pav11, DDT17]. Yet, starting from seminal work of Chawla, Hartline, and Kleinberg [CHK07], a large body of work now proves that simple auctions are in fact approximately optimal in quite general settings [CHMS10, CMS15, HN17, LY13, BILW14, Yao15, RW15, CM16, CZ17, $\mathrm{EFF}^{+}$17b], helping to explain the prevalence of simple auctions in practice. Still, it would be a reach to claim that this agenda is convincingly resolved.

In particular, the thought of settling for $50 \%$ (or even $90 \%$ ) of the optimal achievable revenue may be a non-starter for highstakes auctions. Indeed, there are no hard constraints forcing the auctioneer to use a simple auction. Still, Prior-independent auctions are desirable since they don't require the auctioneer to understand the population from which consumers are drawn. Deterministic and Dominant Strategy Truthful auctions are desirable because consumers' strategic behavior is easier to predict. Computationally tractable auctions are desirable because they can be efficiently found. On the other hand, it is hard to imagine that auctioneers stand a hard line on simplicity if additional market research or outsourcing computation would increase revenues, even modestly.

The resource augmentation paradigm takes a different view: spend effort recruiting additional bidders rather than carefully designing a superior auction. We are therefore interested in answering the following question: for a given auction setting, how many additional bidders are necessary for a simple auction (with additional bidders) to guarantee greater expected revenue than the optimal (without)? Eden et al. term the answer to this question the competition complexity $\left[\mathrm{EFF}^{+} 17 \mathrm{a}\right]$.

This question was first studied in a seminal work by Bulow and Klemperer in the context of single-item auctions [BK96]. Remarkably, they show that just a single additional bidder suffices for the second-price auction to guarantee greater expected revenue than Myerson's optimal auction [Mye81] (without the additional bidder), subject to a technical condition on the population called regularity. For multi-item auctions, similar results have even more bite, as the optimal multi-item auction is considerably more complex than Myerson's (which is deterministic, dominant strategy truthful, and computationally tractable, but not prior-independent). Our main result is optimal bounds on the competition complexity for the core setting of additive bidders with independent items. Specifically, 
Main Result: The competition complexity of $n$ bidders with additive values over $m$ independent items is at most $n(2+\ln (1+m / n))$, and also $9 \sqrt{n m}$. When $n \leq m$ the first bound is tight (up to constant factors). When $n \geq m$, the second bound is tight (up to constant factors) for any argument that starts from the benchmark introduced in $\left[\mathrm{EFF}^{+} 17 \mathrm{a}\right]$.

\subsection{Brief Technical Overview}

Formally, we consider $n$ bidders drawn independently from a distribution $D$. We study the now-standard setting of additive bidders over $m$ independent items: each bidder's value $v_{j}$ for item $j$ is drawn independently from some single-variate distribution $D_{j}$, and her value for a set $S$ of items is $\sum_{j \in S} v_{j}$. The simple mechanism we study is to sell the items separately, either via the second-price auction in the case of regular distributions, or Myerson's optimal single-item auction in the general case. ${ }^{1}$ Observe that, since the bidders are additive and values are independent, selling the items separately is really just $m$ separate single-item problems. We are interested in understanding the minimum $c(n, m)$ such that selling separately to $n+c(n, m)$ bidders drawn from $D$ yields greater expected revenue than the optimal mechanism with $n$ bidders drawn from $D$ for any $D=\times{ }_{j=1}^{m} D_{j}$.

Our approach starts from the benchmark proposed in $\left[\mathrm{EFF}^{+} 17 \mathrm{a}\right]$. That is, Eden et al. propose an upper bound on the optimal achievable revenue with $n$ bidders drawn from $D$ via the duality framework of [CDW16]. ${ }^{2}$ We defer a definition of this benchmark to Section 2.2: it defines a Virtual Value $\Phi_{j}\left(\vec{v}_{i}\right)$ of a bidder with values $\vec{v}_{i}$ for item $j$, and upper bounds the optimal expected revenue with $\mathbb{E}\left[\sum_{j} \max _{i \in[n]}\left\{\Phi_{j}\left(\vec{v}_{i}\right)\right\}\right]$. We defer most details to the technical sections, but briefly note that at this point, our analysis diverges from prior work. Eden et al. use an elegant coupling argument to connect this benchmark to the expected revenue of selling separately with additional bidders $\left[\mathrm{EFF}^{+} 17 \mathrm{a}\right]$. The high-level distinction in our approach is a significantly more in-depth analysis of this benchmark. In particular, our analysis makes more extensive use of Myersonian virtual value theory (Sections 3 and 4), which reduces the problem to questions purely regarding whether various methods of drawing correlated values from $[0,1]$ stochastically dominate one another (Section 5).

\subsection{Connection to Related Works}

The two works most directly related to ours are $\left[\mathrm{EFF}^{+} 17 \mathrm{a}\right]$ and [FFR18]. The one-sentence distinction between our results and these is that we strictly improve their main results regarding selling separately to additive bidders with independent items, but do not address alternative settings. For example, this paper contains no results beyond additive bidders (considered in $\left[\mathrm{EFF}^{+} 17 \mathrm{a}\right]$ ), or results for mechanisms aside from selling separately (considered in [FFR18]).

\footnotetext{
${ }^{1}$ For irregular distributions, it is known that no guarantees are possible with prior independence, even for a single item. The example to have in mind is a distribution with a point mass at $p$ with probability $1 / p$ and 0 otherwise: as $p \rightarrow \infty$, any auction that achieves revenue close to optimum must sell the item to the bidder with value $p$ for price close to $p$ whenever there is exactly one. It is impossible to have a single auction that does this for all $p$.

${ }^{2}$ We note that this upper bound can also be derived without duality using techniques of [CMS15].
}

"Little $n$ Regime": For $n$ additive bidders with $m=\Omega(n)$ independent items, Eden et al. $\left[\mathrm{EFF}^{+} 17 \mathrm{a}\right]$ prove a competition complexity bound of $n+2(m-1)$. Feldman et al. [FFR18] prove that selling separately to $O(n \ln (m / n) / \varepsilon)$ additional buyers exceeds a $(1-\varepsilon)$ fraction of the optimal revenue (without the additional buyers). Our main result essentially achieves the greatly improved bound of [FFR18] (and improves it further) without losing any revenue: we prove a competition complexity bound of $n(2+\ln (1+m / n))$. This guarantee is tight up to constant factors (and remains tight even if one is willing to lose an $\varepsilon$ fraction), due to a lower bound of [FFR18].

"Big $n$ Regime": For $n$ additive bidders with $m=o(n)$ independent items, Eden et al. $\left[\mathrm{EFF}^{+} 17 \mathrm{a}\right]$ prove a competition complexity bound of $n+2(m-1)$. Feldman et al. [FFR18] prove that for any $\varepsilon$, there exists a constant $\delta(\varepsilon)$ such that if $n \geq m / \delta(\varepsilon)$, selling separately (without any additional bidders) achieves a $(1-\varepsilon)$ fraction of the optimal revenue. Our main result improves the guarantee of [ $\mathrm{EFF}^{+} 17 \mathrm{a}$ ] to $9 \sqrt{\mathrm{nm}}$ and also implies the result of [FFR18] (with $\left.\delta(\varepsilon)=\varepsilon^{2} / 81\right)$. Note in particular that any sublinear competition complexity bound implies the [FFR18] result for a different $\delta(\cdot)$, but that linear bounds do not. So our improvement from linear to sublinear is significant in this regard. Moreover, we show in the full version of the paper that this is tight (up to constant factors) for any approach starting from the benchmark proposed in $\left[\mathrm{EFF}^{+} 17 \mathrm{a}\right]$. We further show (also in the full version) that there does not exist any function only of $m$ upper bounding the competition complexity: as $n \rightarrow \infty$ the competition complexity approaches $\infty$ as well (at a rate of at least $\Omega(\ln n)$ ).

Other works that study the competition complexity of auctions include seminal work of Bulow and Klemperer, who study the $m=1$ case, work of Liu and Psomas (who study the competition complexity of dynamic auctions) and Roughgarden et al. (who study the unit-demand setting) [BK96, LP18, RTCY12]. These works are thematically related, but both the results and techniques have little overlap.

Some of the aforementioned works which prove approximation guarantees for simple mechanisms use similar techniques to derive a tractable benchmark that upper bound on the achievable revenue [CHK07, CHMS10, CMS15, HN17, LY13, BILW14, Yao15, RW15, CM16, CZ17, $\mathrm{EFF}^{+}$17b]. However, it is worth noting that all of these works proceed by immediately splitting the benchmark into multiple simpler terms and finding approximately optimal mechanisms to cover each term separately. The best of those mechanism guarantees approximate optimality to revenue. This greatly simplifies analysis, at the cost of an additional constant factor. Because competition complexity results target the full original revenue, losing this initial constant factor can make future analysis impossible. As a result, while benchmarks may be shared by these lines of work, analysis of the benchmarks is often quite different.

Finally, it is worth noting that recent work follows two approaches to derive revenue upper bounds in these works. Some (including this paper) use virtual value theory [CHK07, CHMS10, RTCY12, CMS15, CDW16, CZ17, $\mathrm{EFF}^{+} 17 \mathrm{a}, \mathrm{EFF}^{+} 17 \mathrm{~b}$, LP18, FLLT18]. Others use a more direct probabilitistic approach [HN17, LY13, BILW14, Yao15, RW15, CM16, BGN17, FFR18]. For the most part, similar approximation guarantees are achievable through both approaches. With respect to these lines of work, 
our results (which yield exact competition complexity bounds) in comparison to those of [FFR18] (which lose an arbitrarily small $\varepsilon$ ) suggest the virtual value approach may be desirable if one cares about small losses.

\subsection{Roadmap}

Our main result tightly characterizes the competition complexity in the little $n$ regime, and tightly characterizes the competition complexity in the big $n$ regime among proofs which use the same benchmark as $\left[\mathrm{EFF}^{+} 17 \mathrm{a}\right]$.

In Section 2, we provide the necessary preliminaries surrounding the benchmark of $\left[\mathrm{EFF}^{+} 17 \mathrm{a}\right]$ and virtual value theory. In Section 3 we provide a near-complete proof of our results when $n=1$ as a warm-up. In Section 4, we analyze the benchmark and reduce the analysis to proving stochastic dominance of certain correlated random variables drawn from $[0,1]$. In Section 5 we prove the required claims regarding stochastic dominance (which at this point are purely mathematical claims and no longer reference auctions).

\section{NOTATION AND PRELIMINARIES}

We consider a setting with $n$ i.i.d. bidders with additive valuations over $m$ independent items. That is, there are single-variate distributions $D_{j}$ for all $j \in[m]$, and bidder $i$ 's value $v_{j}$ for item $j$ is drawn independently from $D_{j}$. Bidder $i$ 's value for the bundle $S$ is just $\sum_{j \in S} v_{j}$. We will use the following notation:

- $\operatorname{Rev}_{n}(D)$ to denote the revenue of the optimal (possibly randomized) Bayesian Incentive Compatible ${ }^{3}$ mechanism when played by $n$ bidders whose values for $m$ items are drawn from $D$. In our setting, we will always have $D=$ $\times_{j} D_{j}$ for some single-variate distributions $D_{j}$.

- $\mathrm{VCG}_{n}(D)$ to denote the revenue achieved by the VCG mechanism when played by $n$ bidders whose values for $m$ items are drawn from $D$. In our setting, the VCG mechanism simply runs a second-price auction on each item separately with no reserve.

- $\operatorname{SRev}_{n}(D)$ to denote the revenue achieved by Myerson's mechanism run separately on each item, when played by $n$ bidders whose values for $m$ items are drawn from $D$. Note that for all $n$ and distributions $D$ over additive valuations, $\operatorname{SREv}_{n}(D) \geq \operatorname{VCG}_{n}(D)$.

\subsection{Myerson's Lemma, Bulow-Klemperer, and Virtual Values}

Here, we briefly recap basic facts about the theory of virtual values due to Myerson [Mye81]. We include some proofs and sketches in the appendix of the full version of the paper, and refer the reader to [Har11] (Definition 3.11) for a deeper treatment of these concepts (or [CDW16], Definition 8 for discrete distributions). Note that much of the theory extends to independent (but non-i.i.d.) bidders with slightly more complex statements. As we only consider i.i.d. bidders, we omit the extra notation. Below, when we write $X^{+}$for a random variable $X$, we mean $\max \{X, 0\}$.

${ }^{3} \mathrm{~A}$ mechanism is Bayesian Incentive Compatible if it is in every bidder's interest to bid truthfully, conditioned on all other bidders bidding truthfully as well. That is, assuming that all other bidders submit bids drawn from $D_{-i}$, bidder $i$ best responds by bidding their true values.
Definition 1 (Virtual Values and Ironing [Mye81]). For a continuous single-variate distribution with $C D F F(\cdot)$ and PDF $f(\cdot)$, the virtual valuation function $\varphi_{F}(\cdot)$ satisfies $\varphi_{F}(v)=v-\frac{1-F(v)}{f(v)}$. If $\varphi_{F}(\cdot)$ is monotone non-decreasing, $F$ is said to be regular. If not, $\bar{\varphi}_{F}(\cdot)$ is the ironed virtual value function, and is monotone nondeceasing (see [Har11] for a formal definition). When $F$ is regular, $\bar{\varphi}_{F}(\cdot)=\varphi_{F}(\cdot)$.

Theorem 1 ([Mye81]). Let $D$ be any single-variate distribution. Then for all $n$ :

$$
\begin{aligned}
& \operatorname{SREV}_{n}(D)=\operatorname{REV}_{n}(D)=\mathbb{E}_{\vec{v} \leftarrow D^{n}}\left[\left(\bar{\varphi}_{D}\left(\max _{i \in[n]}\left\{v_{i}\right\}\right)\right)^{+}\right] \\
& \operatorname{VCG}_{n}(D)=\mathbb{E}_{\vec{v} \leftarrow D^{n}}\left[\left(\varphi_{D}\left(\max _{i \in[n]}\left\{v_{i}\right\}\right)\right)\right] .
\end{aligned}
$$

FACT 1. For any single-variate distribution $D$, and any value $v$, let $D_{\geq v}$ denote the distribution $D$ conditioned on exceeding $v$. Then $v=\mathbb{E}_{w \leftarrow D_{\geq v}}[\varphi(w)] \leq \mathbb{E}_{w \leftarrow D_{\geq v}}[\bar{\varphi}(w)]$.

Finally, we recall the seminal result of Bulow and Klemperer [BK96]:

THEOREM 2 ([BK96]). For any regular single-variate distribution $D, V C G_{n+1}(D) \geq \operatorname{REV}_{n}(D)$.

\subsection{Duality Benchmarks}

Here we state an upper bound on $\operatorname{Rev}_{n}(D)$ when $D$ is additive over independent items. The bound is derived using the duality framework of Cai et al. [CDW16], and first used by Eden et al. [EFF ${ }^{+} 17 \mathrm{a}$ ] (it is also possible to derive this particular bound without duality [CMS15]). When referring to this benchmark in text, we call it the EFFTW benchmark. Parsing the benchmark requires additional notation:

- $v_{i j}$ denotes the value of bidder $i$ for item $j$.

- $D_{j}$ denotes the marginal distribution of item $j$. We use $\bar{\varphi}_{j}(\cdot)$ to denote $\bar{\varphi}_{D_{j}}(\cdot)$.

- For a variable $X$, if $X$ has no point-masses, then we simply define $F(x)=\operatorname{Pr}[X<x]=\operatorname{Pr}[X \leq x]$. If $X=x$ with strictly positive probability, then we define $F(x)$ to be a random variable drawn uniformly from the interval $[\operatorname{Pr}[X<x], \operatorname{Pr}[X \leq x]]$. Importantly, note that the random variable $F(X)$ is drawn uniformly from $[0,1]$ for any random variable $X$.

- $F_{j}(x)$ denotes $F(x)$ with respect to random variable $X$ drawn from $D_{j}$.

- For a distribution $D:=\times_{j} D_{j}$, we partition the space $\mathbb{R}_{+}^{m}$ into $m$ disjoint regions. For each $j \in[m]$, we define $R_{j}:=$ $\left\{\vec{v}_{i} \in \mathbb{R}_{+}^{m} \mid F_{j}\left(v_{i j}\right)>F_{k}\left(v_{i k}\right) \forall k \neq j\right\}$. That is, $\vec{v}_{i}$ is in region $R_{j}$ if item $j$ has the highest quantile. Observe that his partition may be randomized if $D$ has point masses (and is deterministic with probability 1 if $D$ has no point masses).

THeOREM 3 ([CDW16, $\left.\left.\mathrm{EFF}^{+} 17 \mathrm{~A}\right]\right)$. Let $D$ be additive over $m$ independent items. Then:

$$
\begin{gathered}
\operatorname{REV}_{n}(D) \leq \\
\sum_{j=1}^{m} \mathbb{E}_{\vec{v} \leftarrow D^{n}}\left[\max _{i \in[n]}\left\{\bar{\varphi}_{j}\left(v_{i j}\right)^{+} \cdot \mathbb{I}\left(\vec{v}_{i} \in R_{j}\right)+v_{i j} \cdot \mathbb{I}\left(\vec{v}_{i} \notin R_{j}\right)\right\}\right]
\end{gathered}
$$


If we think of the Virtual Value of bidder $i$ for item $j$ as equal to Myerson's ironed virtual value, $\bar{\varphi}_{j}\left(v_{i j}\right)^{+}$, if item $j$ has the highest quantile in $\vec{v}_{i}$, and equal to the value, $v_{i j}$, if not, then Theorem 3 claims that the expected revenue of the optimal mechanism does not exceed the sum over all items of the expected maximum virtual value for that item. Theorem 3 is an application of Corollary 28 in [CDW16], together with the observation that our defined regions are upwards-closed.

\section{WARM-UP: SINGLE BIDDER}

In this section, we illustrate one portion of our improved anlaysis via the single bidder setting. This will also help identify one significant point of departure from $\left[\mathrm{EFF}^{+} 17 \mathrm{a}\right]$. Observe that the EFFTW benchmark simplifies significantly for a single bidder, as there is only one element of $[n]$, and the benchmark simply sums the virtual value of the item with the highest quantile plus the values of all other items.

\subsection{Brief Recap of $\left[\mathrm{EFF}^{+} 17 \mathrm{a}\right]$}

The main idea in the single-bidder approach of $\left[\mathrm{EFF}^{+} 17 \mathrm{a}\right]$ is to couple draws of $m$ bidders for item $j$ with draws of a single bidder for $m$ items via their quantiles. Specifically, they observe the following: consider fixed quantiles $q_{1}, \ldots, q_{m}$ drawn independently. and uniformly from $[0,1]$.

- Benchmark Analysis: Use the quantiles drawn to determine values for each of $m$ items. If $q_{j}$ is the largest quantile drawn, then item $j$ contributes $\bar{\varphi}_{j}\left(F_{j}^{-1}\left(q_{j}\right)\right)^{+}$to the benchmark. If $q_{j}$ is not the largest quantile drawn, then item $j$ contributes $F_{j}^{-1}\left(q_{j}\right)$ to the benchmark.

- VCG Analysis: Use the quantiles drawn to determine values of each of $m$ bidders for item $j$. If $q_{j}$ is the largest quantile drawn, then bidder $j$ contributes $\bar{\varphi}_{j}\left(F_{j}^{-1}\left(q_{j}\right)\right)$ to the virtual surplus of VCG. If $q_{j}$ is not the largest quantile drawn, then some other bidder wins the item and pays at least $F_{j}^{-1}\left(q_{j}\right)$, so at least $F_{j}^{-1}\left(q_{j}\right)$ is contributed by some bidder $\neq j$ to the revenue.

The above reasoning is not far from a formal proof that $\operatorname{SREv}_{m}(D) \geq$ $\operatorname{Rev}_{1}(D)$. Some care is required to make sure Theorem 1 is applied correctly (since we wish to count bidder $j$ 's contribution to the revenue of VCG using her ironed virtual value but the other bidders' contributions directly via payments), but the above reasoning is the key step. The main idea is that if we couple the quantiles drawn for the benchmark with quantiles drawn for selling just item $j$, then the revenue achieved from selling just item $j$ to $m$ bidders drawn from $D_{j}$ exceeds the contribution of item $j$ to the benchmark for all quantiles drawn.

\subsection{Our Analysis}

The main challenge that the previous analysis overcomes is the following: the contribution of item $j$ to the benchmark is sometimes in the form of a virtual value, and sometimes in the form of a value. There is no "natural" random variable that takes exactly this form, and it is tricky to analyze directly. So the previous analysis finds a clever way to "recreate" it using this coupling argument. Unfortunately though, direct coupling arguments like this should not hope to prove a competition complexity better than $m-1$, as there are $m$ random variables that need to be coupled.

Our approach instead is to reason about the contribution of item $j$ to the benchmark exclusively in terms of virtual values, using Fact 1. Specifically, consider the following proposition, which rewrites the contribution of item $j$ to the benchmark. Below, $X_{L}(1, m)$ denotes the following random variable ${ }^{4}$ : first, draw one quantile $X_{1,1}$ uniformly at random from $[0,1]$. Then, draw $m-1$ quantiles uniformly at random from $[0,1]$ and label them $Y_{1, m-1}$ through $Y_{m-1, m-1}$. If $X_{1,1}>Y_{\ell, m-1}$ for all $\ell$, then set $X_{L}(1, m)=X_{1,1}$. Otherwise, let $\ell^{*}$ denote a uniformly random element from $\left\{\ell \mid Y_{\ell, m-1}>X_{1,1}\right\}$ and set $X_{L}(1, m)=Y_{\ell^{*}, m-1}$.

Proposition 1. For all $D=\times_{j} D_{j}$ and all items $j$,

$$
\begin{gathered}
\mathbb{E}_{\vec{v} \leftarrow D}\left[\bar{\varphi}_{j}\left(v_{j}\right)^{+} \cdot \mathbb{I}\left(\vec{v} \in R_{j}\right)+v_{j} \cdot \mathbb{I}\left(\vec{v} \notin R_{j}\right)\right] \\
\leq \mathbb{E}\left[\bar{\varphi}_{j}\left(F_{j}^{-1}\left(X_{L}(1, m)\right)\right)^{+}\right] .
\end{gathered}
$$

Proof. The main idea is to get a lot of mileage from Fact 1: ideally, any time $\vec{v} \notin R_{j}$, rather than contribute $v_{j}$ to the benchmark, we will contribute the virtual value of a random draw from $D_{j}$ conditioned on exceeding $v_{j}$. To begin, let's couple quantiles drawn for the benchmark with quantiles drawn for the experiment defining $X_{L}(1, m)$ so that $X_{1,1}=F_{j}\left(v_{j}\right)$ and $Y_{\ell, m-1}=F_{\ell}\left(v_{\ell}\right)$ for $\ell<m, \ell \neq j$, and $Y_{j, m-1}=F_{m}\left(v_{m}\right)$ (if $j \neq m$, otherwise there is no $Y_{m, m-1}$ to define). Observe that indeed the quantiles are all drawn independently and uniformly from $[0,1]$. Moreover, we have:

- Whenever $\vec{v} \in R_{j}, X_{L}(1, m)=X_{1,1}=F_{j}\left(v_{j}\right)$. Therefore, we conclude that:

$$
\begin{gathered}
\mathbb{E}_{\vec{v} \leftarrow D}\left[\bar{\varphi}_{j}\left(v_{j}\right)^{+} \cdot \mathbb{I}\left(\vec{v} \in R_{j}\right)\right] \\
=\mathbb{E}\left[\bar{\varphi}_{j}\left(F_{j}^{-1}\left(X_{L}(1, m)\right)^{+} \cdot \mathbb{I}\left(X_{L}(1, m)=X_{1,1}\right)\right] .\right.
\end{gathered}
$$

- Conditioned on $\vec{v} \notin R_{j}, X_{L}(1, m)$ is a uniformly random sample from $\left[X_{1,1}, 1\right]$. This is because there is some strictly positive number of $\ell$ s such that $Y_{\ell, m-1}>X_{1,1}$. Conditioned on being $>X_{1,1}$, each such value is drawn uniformly from $\left[X_{1,1}, 1\right]$. And then $X_{L}(1, m)$ picks one of them uniformly at random. Using Fact 1, we therefore conclude that:

$$
\begin{aligned}
& \mathbb{E}_{\vec{v} \leftarrow D}\left[v_{j} \cdot \mathbb{I}\left(\vec{v} \notin R_{j}\right)\right] \\
\leq & \mathbb{E}_{\vec{v} \leftarrow D}\left[\mathbb{E}_{x \leftarrow D_{j, \geq v_{j}}}\left[\bar{\varphi}_{j}(x)\right] \cdot \mathbb{I}\left(\vec{v} \notin R_{j}\right)\right] \\
\leq & \mathbb{E}\left[\bar{\varphi}_{j}\left(F_{j}^{-1}\left(X_{L}(1, m)\right)\right) \cdot \mathbb{I}\left(X_{L}(1, m) \neq X_{1,1}\right)\right] .
\end{aligned}
$$

It is now easy to see that the left-hand sides of the two equations sum together to yield item $j$ 's contribution to the benchmark, while the two right-hand sides sum together to yield (at most) $\mathbb{E}\left[\bar{\varphi}_{j}\left(F_{j}^{-1}\left(X_{L}(1, m)\right)\right)^{+}\right]$, proving the proposition.

Proposition 1 gives an upper bound on the contribution of item $j$ to the benchmark written as the expectation of a virtual value of some distribution $\left(F_{j}^{-1}\left(X_{L}(1, m)\right)\right)$. This is convenient because we can write the revenue achieved by using Myerson's optimal auction for selling item $j$ to $1+c$ bidders as the expectation of a virtual value of another distribution (the maximum of $1+c$ draws from $D_{j}$ ). Therefore, if we can relate these two distributions (for

${ }^{4} X_{L}(n, m)$ relates to Little $n$ Benchmark Experiment defined (Section 5). 
instance, by proving that one stochastically dominates the other), we can relate these two expectations. Below, let $X_{S}(1, c)$ denote the maximum of $1+c$ i.i.d. draws from the uniform distribution on $[0,1] .^{5}$

Corollary 1. If $X_{S}(1, c)$ stochastically dominates $X_{L}(1, m)$, then for all $D$ that are additive overm independent items, $\operatorname{SREV}_{1+c}(D) \geq$ $\operatorname{REV}_{1}(D)$.

Proof. Observe first that by Theorem 1 we have:

$$
\begin{aligned}
\operatorname{SREV}_{1+c}(D) & =\sum_{j=1}^{m} \mathbb{E}_{\vec{x} \leftarrow D_{j}^{1+c}}\left[\bar{\varphi}_{j}\left(\max _{i \in[1+c]}\left\{x_{i}\right\}\right)^{+}\right] \\
& =\sum_{j=1}^{m} \mathbb{E}\left[\bar{\varphi}_{j}\left(F_{j}^{-1}\left(X_{S}(1, c)\right)^{+}\right]\right.
\end{aligned}
$$

By Proposition 1 (and Theorem 3), we have:

$$
\begin{aligned}
\operatorname{REV}_{1}(D) & \leq \sum_{j=1}^{m} \mathbb{E}_{\vec{v} \leftarrow D}\left[\bar{\varphi}_{j}\left(v_{j}\right)^{+} \cdot \mathbb{I}\left(\vec{v} \in R_{j}\right)+v_{j} \cdot \mathbb{I}\left(\vec{v} \notin R_{j}\right)\right] \\
& \leq \sum_{j=1}^{m} \mathbb{E}\left[\bar{\varphi}_{j}\left(F_{j}^{-1}\left(X_{L}(1, m)\right)\right)^{+}\right] .
\end{aligned}
$$

Observe that $\bar{\varphi}_{j}(\cdot)$ is a monotone non-decreasing function, and $F_{j}^{-1}$ is also monotone non-decreasing. As such, if $X_{S}(1, c)$ stochastically dominates $X_{L}(1, m), \bar{\varphi}_{j}\left(F_{j}^{-1}\left(X_{S}(1, c)\right)\right)$ stochastically dominates $\bar{\varphi}_{j}\left(F_{j}^{-1}\left(X_{L}(1, m)\right)\right)$, which allows us to conclude that

$$
\sum_{j} \mathbb{E}\left[\bar{\varphi}_{j}\left(F_{j}^{-1}\left(X_{S}(1, c)\right)^{+}\right] \geq \sum_{j=1}^{m} \mathbb{E}\left[\bar{\varphi}_{j}\left(F_{j}^{-1}\left(X_{L}(1, m)\right)\right)^{+}\right]\right.
$$

. Therefore, we may conclude that if $X_{S}(1, c)$ stochastically dominates $X_{L}(1, m), \operatorname{SRev}_{1+c}(D) \geq \operatorname{Rev}_{1}(D)$.

At this point, we've reduced the problem of deriving competition complexity upper bounds to a purely mathematical problem relating stochastic dominance of $X_{S}(1, c)$ and $X_{L}(1, m)$. The proof of this claim for $n=1$ is not an especially instructive special case, so we defer the final step to Section 5. So we wrap up our warm-up by citing Theorem 8:

Corollary 2 (of Theorem 8). When $c \geq 2+\ln (m+1)$, $X_{S}(1, c)$ stochastically dominates $X_{L}(1, m)$.

Theorem 4. Let $D$ be a distribution that is additive over $m$ independent items. Then $\operatorname{SREV}_{2+\ln (m+1)}(D) \geq \operatorname{REV}_{1}(D) .{ }^{6}$ If each $D_{j}$ is regular, then also $V C G_{3+\ln (m+1)}(D) \geq \operatorname{REV}_{1}(D)$.

Proof. Theorem 3 upper bounds $\operatorname{Rev}_{1}(D)$ with the EFFTW benchmark. Proposition 1 further upper bounds the EFFTW benchmark with $\sum_{j} \mathbb{E}\left[\bar{\varphi}_{j}\left(F_{j}^{-1}\left(X_{L}(1, m)\right)\right)^{+}\right]$, which is the sum over all items of the expected virtual value of a quantile drawn from $X_{L}(1, m)$. Corollary 1 argues that if $X_{L}(1, m)$ is stochastically dominated by $X_{S}(1, c)$ (the maximum of $c+1$ i.i.d. draws uniformly from $[0,1])$, then we may replace $X_{L}(1, m)$ with $X_{S}(1, c)$ in the upper bound, which is exactly $\operatorname{SREv}_{1+c}(D)$. Finally, Corollary 2

\footnotetext{
${ }^{5} X_{S}(n, c)$ relates to SRev Experiment defined (Section 5).

${ }^{6}$ This result implies a minor improvement in the approximation ratio of SREV vs REV in Theorem 1 of [BILW14], from $3+\ln (m)$ to $2+\ln (m+1)$.
}

claims that indeed $X_{S}(1, c)$ stochastically dominates $X_{L}(1, m)$ when $c \geq 2+\ln (m+1)$ (and the final +1 when each $D_{j}$ is regular comes from going from SREv to VCG using Theorem 2).

This concludes our exposition for a single bidder. Above we introduced one new idea which departs from prior work: instead of directly treating the benchmark which involves both values and virtual values, rewrite the benchmark to involve only virtual values and reduce the problem to purely mathematical questions about stochastic dominance of $X_{L}(1, m)$ and $X_{S}(1, c)$.

\section{MULTIPLE BIDDERS}

In this section, we overview our approach for the general case. The key simplifying feature of the single-bidder case that allowed us to isolate one key idea is that for each item $j$, that item has the highest quantile or it doesn't. In the multi-bidder case, there are multiple bidders, some of whom will have their highest quantile for item $j$, some of whom won't. So we must actually engage with the " $\max _{i \in[n]}$ " in the benchmark. Our approach will be different depending on whether $n$ is big or little relative to $m$. We begin with the little $n$ case as it is more similar to the single-bidder case.

\subsection{Part One: When $n \leq m$}

Our key step is conceptually similar to Proposition 1, but the random variables involved are necessarily more complex. We first make the following observation (also made in $\left.\left[\mathrm{EFF}^{+} 17 \mathrm{a}\right]\right)$. Below, $v_{(\ell) j}$ denotes the $\ell^{\text {th }}$ highest value for item $j$ (among all bidders). Also for any fixed $j, \vec{v}_{(i)}$ denotes the value vector of the bidder who has the $i^{\text {th }}$ highest value for item $j$. All omitted proofs can be found in the appendix of the full version of the paper.

ObServation 1. For all items $j$,

$$
\begin{gathered}
\mathbb{E}_{\vec{v} \leftarrow D^{n}}\left[\max _{i \in[n]}\left\{\bar{\varphi}_{j}\left(v_{i j}\right)^{+} \cdot \mathbb{I}\left(\vec{v}_{i} \in R_{j}\right)+v_{i j} \cdot \mathbb{I}\left(\vec{v}_{i} \notin R_{j}\right)\right\}\right] \\
\leq \mathbb{E}_{\vec{v} \leftarrow D^{n}}\left[\max \left\{v_{(1) j} \cdot \mathbb{I}\left(\vec{v}_{(1)} \notin R_{j}\right), \bar{\varphi}_{j}\left(v_{(1) j}\right), v_{(2) j}\right\}\right] .
\end{gathered}
$$

Next, we want to rewrite the right-hand side above using random variables similar to $X_{L}(1, m)$. This time, let $X_{L}^{\prime}(n, m)$ denote the following random variable: first, draw $n$ quantiles $X_{1, n}, \ldots, X_{n, n}$ independently and uniformly at random from $[0,1]$. Relabel them so that $X_{(1), n} \geq \ldots \geq X_{(n), n}$. Then, draw $m-1$ quantiles uniformly at random from $[0,1]$ and label them $Y_{1, m-1}$ through $Y_{m-1, m-1}$. If $X_{(1), n}>Y_{\ell, m-1}$ for all $\ell$, then set $X_{L}^{\prime}(n, m)=$ $X_{(1), n}$. Otherwise, let $\ell^{*}$ be a uniformly random element from $\left\{\ell \mid Y_{\ell, m-1}>X_{(1), n}\right\}$ and set $X_{L}^{\prime}(n, m)=Y_{\ell^{*}, m-1}$.

Proposition 2. For all $D=\times_{j} D_{j}$, and all items $j$ :

$$
\begin{aligned}
& \mathbb{E}_{\vec{v} \leftarrow D^{n}}\left[\max \left\{v_{(1) j} \cdot \mathbb{I}\left(\vec{v}_{(1)} \notin R_{j}\right), \bar{\varphi}_{j}\left(v_{(1) j}\right), v_{(2) j}\right\}\right] \\
& \leq \mathbb{E}\left[\max \left\{\bar{\varphi}_{j}\left(F_{j}^{-1}\left(X_{L}^{\prime}(n, m)\right)\right), F_{j}^{-1}\left(X_{(2), n}\right)\right\}\right] .
\end{aligned}
$$

Proposition 2 helps us replace any instances of $v_{(1) j}$ in the benchmark with a randomly drawn virtual value, but we still need to do the same for $v_{(2) j}$ (which so far has essentially just been rewritten as $\left.F_{j}^{-1}\left(F_{j}\left(v_{(2) j}\right)\right)\right)$. Now, let $W_{2, n}$ be a uniformly random draw from $\left[X_{(2), n}, 1\right]$, and define $X_{L}(n, m)=\max \left\{X_{L}^{\prime}(n, m), W_{2, n}\right\}$. By making use of Fact 1, we can conclude: 
Corollary 3.

$$
\begin{aligned}
\mathbb{E}[\max & \left.\left\{\bar{\varphi}_{j}\left(F_{j}^{-1}\left(X_{L}^{\prime}(n, m)\right)\right), F_{j}^{-1}\left(X_{(2), n}\right)\right\}\right] \\
& \leq \mathbb{E}\left[\bar{\varphi}_{j}\left(F_{j}^{-1}\left(X_{L}(n, m)\right)\right)\right] .
\end{aligned}
$$

Now, we are nearly ready to wrap up the $n \leq m$ case. Similarly to the single-bidder case, define $X_{S}(n, c)$ to be the maximum of $n+c$ i.i.d. draws uniformly form $[0,1]$.

Corollary 4. If $X_{S}(n, c)$ stochastically dominates $X_{L}(n, m)$, then $\operatorname{SREV}_{n+c}(D) \geq \operatorname{REV}_{n}(D)$. If each $D_{j}$ is regular, then $V C G_{n+c}(D) \geq$ $\operatorname{REV}_{n}(D)$.

Finally, Theorem 8 claims that when $c \geq n \cdot(2+\ln (1+m / n))$, $X_{S}(n, c)$ indeed stochastically dominates $X_{L}(n, m)$. Combining Corollary 4 with Theorem 8 therefore concludes:

THEOREM 5. For all $D$ that are additive over $m$ independent items, $\operatorname{SREV}_{n+n \cdot(2+\ln (1+m / n))}(D) \geq \operatorname{REV}_{n}(D)$. If each marginal of $D_{j}$ is regular, then $V C G_{n+n \cdot(2+\ln (1+m / n))}(D) \geq \operatorname{REV}_{n}(D)$.

When $n \leq m$, this is tight up to constant factors, due to a lower bound of [FFR18] (see the appendix of the full version for the construction). But when $n \geq m$, this is still linear in $n$. We therefore provide an alternative argument in the following section which achieves the optimal (up to constant factors) competition complexity that is achievable starting from the EFFTW benchmark of $\Theta(\sqrt{n m})$.

\subsection{Part Two: When $n \geq m$}

At a high level, the main difference between how we should analyze the $n \leq m$ case and the $n \geq m$ is as follows: Observation 1 immediately upper bounds the EFFTW by upper bounding $\bar{\varphi}_{j}\left(v_{(2) j}\right)^{+} \cdot \mathbb{I}\left(\vec{v}_{(2)} \in R_{j}\right)+v_{(2) j} \cdot \mathbb{I}\left(\vec{v}_{(2)} \notin R_{j}\right)$ with $v_{(2) j}$. When $n \leq m$, this upper bound is unlikely to be much of a relaxation, because it's likely that $v_{(1)} \notin R_{j}$ anyway. But when $n \gg m$, we're extremely unlikely to have $v_{(1)} \notin R_{j}$, and this upper bound is wasteful. Indeed, this step is what limits the analysis in $\left[\mathrm{EFF}^{+} 17 \mathrm{a}\right]$ to $\Omega(n)$. The first step for the $n \geq m$ case is to address this.

Proposition 3. For all items $j$, all $\ell \in[n]$, and all distributions $D$ that are additive over independent items:

$$
\begin{gathered}
\mathbb{E}_{\vec{v} \leftarrow D^{n}}\left[\max _{i \in[n]}\left\{\bar{\varphi}_{j}\left(v_{i j}\right)^{+} \cdot \mathbb{I}\left(\vec{v}_{i} \in R_{j}\right)+v_{i j} \cdot \mathbb{I}\left(\vec{v}_{i} \notin R_{j}\right)\right\}\right] \\
\leq \mathbb{E}_{\vec{w} \leftarrow D_{j}^{n+(m-1)(\ell-1)}}\left[\max \left\{\bar{\varphi}_{j}\left(w_{(1)}\right), w_{(\ell)}\right\}\right] .
\end{gathered}
$$

We now want to take a similar step to the previous case and replace $w_{(\ell)}$ with a randomly drawn virtual value using Fact 1. Here, define the random variable $X_{B}(n, \ell)$ as follows. ${ }^{7}$ First, draw $X_{1, n}, \ldots, X_{n, n}$ independently and uniformly at random from $[0,1]$. Then, randomly draw $W_{\ell, n}$ uniformly from $\left[X_{(\ell), n}, 1\right]$, and set $X_{B}(n, \ell):=\max \left\{X_{(1), n}, W_{\ell, n}\right\}$.

Lemma 1. For any single-dimensional distribution $D$, and any $n^{\prime}$ : $\mathbb{E}_{\vec{w} \leftarrow D^{n^{\prime}}}\left[\max \left\{\bar{\varphi}_{j}\left(w_{(1)}\right), w_{(\ell)}\right\}\right] \leq \mathbb{E}\left[\bar{\varphi}_{j}\left(F_{j}^{-1}\left(X_{B}\left(n^{\prime}, \ell\right)\right)\right)\right]$.

Corollary 5 below follows from Proposition 3 and Lemma 1 with $n^{\prime}:=n+(m-1)(\ell-1)$.

\footnotetext{
${ }^{7} X_{B}(n, \ell)$ relates to Big $n$ Benchmark Experiment defined (Section 5)
}

Corollary 5. If $X_{S}(n, c)$ stochastically dominates $X_{B}(n+(\ell-$ $1)(m-1), \ell)$, then for any $D$ that is additive over $m$ independent items, $\operatorname{SREV}_{n+c}(D) \geq \operatorname{REV}_{n}(D)$. If each marginal $D_{j}$ is regular, then $\operatorname{VCG}_{n+c}(D) \geq \operatorname{REV}_{n}(D)$.

Finally, Theorem 7 states that $X_{S}(n, c)=X_{S}(n+(\ell-1)(m-$ $1), c-(\ell-1)(m-1))$ stochastically dominates $X_{B}(n+(\ell-$ $1)(m-1), \ell)$ whenever $c-(\ell-1)(m-1) \geq \frac{4 n+4(\ell-1)(m-1)}{\ell-1}$. Setting $\ell:=\sqrt{n} m+1$, we get $c \geq \sqrt{n m}+4 \sqrt{n m}+4(m-1)$.

THeOREm 6. For all $D$ that are additive over $m$ independent items, $\operatorname{SREV}_{n+5 \sqrt{n m}+4(m-1)}(D) \geq \operatorname{REV}_{n}(D)$. If each marginal $D_{j}$ is reg-

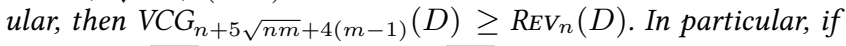
$n \geq m, 5 \sqrt{n m}+4(m-1) \leq 9 \sqrt{n m}$.

\section{STOCHASTIC DOMINANCE VIA ADDITIONAL SAMPLES}

In this section, we consider purely questions about whether one distribution stochastically dominates another (Sections 3 and 4 outline the connection between these problems and our main result). Recall the following ingredients in our experiments:

- $X_{1, n}, \ldots, X_{n, n}$ are $n$ i.i.d. draws from the uniform distribution on $[0,1]$, and then relabeled so that $X_{(1), n} \geq \ldots \geq$ $X_{(n), n}$.

- $Y_{1, m-1}, \ldots, Y_{m-1, m-1}$ are $m-1$ i.i.d. draws from the uniform distribution on $[0,1]$, and then relabeled so that $Y_{(1), m-1} \geq \ldots \geq Y_{(m-1), m-1}$.

- $Z_{1, c}, \ldots, Z_{c, c}$ are $c$ i.i.d. draws from the uniform distribution on $[0,1]$, and then relabeled so that $Z_{(1), c} \geq \ldots \geq$ $Z_{(c), c}$.

- $W_{\ell, n}$ is a random draw from the uniform distribution on $\left[X_{(\ell), n}, 1\right]$.

$\operatorname{SREv} \operatorname{Experiment}(n, c):$ Output $X_{S}(n, c):=\max \left\{X_{(1), n}, Z_{(1), c}\right\}$.

Big $n$ Benchmark Experiment $(n, \ell)$ :

Output $X_{B}(n, \ell):=\max \left\{X_{(1), n}, W_{\ell, n}\right\}$.

Little $n$ Benchmark Experiment $(n, m)$ : Let $j^{*}$ be the largest index such that $Y_{\left(j^{*}\right), m-1}>X_{(1), n}$ (if such a $j^{*}$ exists). If no such $j^{*}$ exists, output $X_{L}(n, m):=\max \left\{X_{(1), n}, W_{2, n}\right\}$. Otherwise, pick an index $j$ uniformly at random from $\left\{1, \ldots, j^{*}\right\}$ and output $\max \left\{Y_{(j), m-1}, W_{2, n}\right\}$.

The main results of this section are as follows:

Theorem 7. When $c \geq 4 n /(\ell-1), X_{S}(n, c)$ stochastically dominates $X_{B}(n, \ell)$.

Theorem 8. When $c \geq n \cdot(2+\ln (1+m / n)), X_{S}(n, c)$ stochastically dominates $X_{L}(n, m)$.

Intuitively, we might expect $X_{S}(n, c)$ to stochastically dominate $X_{B}(n, \ell)$ right around $c=2 n / \ell$. This is because $\mathbb{E}\left[Z_{(1), c}\right]=$ $1-1 /(c+1)$, and $\mathbb{E}\left[W_{\ell, n}\right]=1-\frac{\ell}{2(n+1)}$. Of course, this observation doesn't come close to proving stochastic dominance, especially because $X_{(1), n}$ and $W_{\ell, n}$ aren't independent. But it does give us an idea of the right ballpark to shoot for. The following proposition will be used in the proof of both theorems. 
Proposition 4. Let $c \geq 4 n /(\ell-1)$. Then for all $p, \operatorname{Pr}\left[Z_{(1), c}>\right.$ $\left.p \mid X_{(1), n}<p\right] \geq \operatorname{Pr}\left[W_{\ell, n}>p \mid X_{(1), n}<p\right]$. When $\ell=2$, this can be improved to $c \geq n$.

Before getting into the proof, let's unpack the role of conditioning on $X_{(1), n} . Z_{(1), c}$ and $X_{(1), n}$ are independent, so $\operatorname{Pr}\left[Z_{(1), c}>\right.$ $\left.p \mid X_{(1), n}<p\right]=\operatorname{Pr}\left[Z_{(1), c}>p\right]$. On the other hand, $W_{\ell, n}$ and $X_{(1), n}$ are positively correlated: the lower bound on the range from which $W_{\ell, n}$ is drawn is $X_{(\ell), n}$, which is positively correlated with $X_{(1), n}$. So certainly if we could prove the lemma without the conditioning on $X_{(1), n}<p$, the desired proposition would hold. This approach works for $\ell=2$ (and indeed shows up in our proof as a base case), but without conditioning the conclusion is otherwise false for larger $\ell$.

Proof. The proof will proceed by induction on $n, \ell$. We begin with the base case, $\ell=2$. $Z_{(1), c}$ is easy to reason about: $Z_{(1), c}$ is just the maximum of $c$ i.i.d. draws uniformly from $[0,1]$. So:

$$
\operatorname{Pr}\left[Z_{(1), c}>p \mid X_{(1), n}<p\right]=\operatorname{Pr}\left[Z_{(1), c}>p\right]=1-p^{c} .
$$

Now we turn to $W_{2, n}$. As referenced in the foreword to the proof, for this case the proposition statement holds even without conditioning on $X_{(1), n}<p$. Indeed, observe that without conditioning on $X_{(1), n}<p, X_{(2), n}$ is just the second-highest of $n$ i.i.d. draws uniformly from $[0,1]$, and $W_{2, n}$ is drawn uniformly from $[0,1]$, but conditioned on exceeding $X_{(2), n}$. That is, $W_{2, n}$ is actually identically distributed to $X_{(1), n}$, and is distributed according to the maximum of $n$ i.i.d. draws uniformly from $[0,1]$. Therefore, when $c=n, W_{2, n}$ is identically distributed to $Z_{(1), c}$, and the conclusion holds. That is:

$$
\operatorname{Pr}\left[W_{2, n}>p \mid X_{(1), n}<p\right] \leq \operatorname{Pr}\left[W_{2, n}>p\right]=1-p^{n} .
$$

As such, we have proved the base case (in fact, a slightly stronger claim): for all $n$, and $\ell=2$ when $c \geq n=n /(\ell-1), Z_{(1), c}$ stochastically dominates $W_{2, n}$. Now we turn to the inductive step, which is significantly more involved. As referenced in the foreword, we must take a different approach for larger $\ell$, as the desired claim is false if we remove conditioning on $X_{(1), n}<p$.

To this end, we'll first observe that when $p=1, \operatorname{Pr}\left[Z_{(1), c}>\right.$ $1]=\operatorname{Pr}\left[W_{\ell, n}>1 \mid X_{(1), n}<1\right]=0$, and when $p \rightarrow 0, \operatorname{Pr}\left[Z_{(1), c}>\right.$ $p]=\operatorname{Pr}\left[W_{\ell, n}>p \mid X_{(1), n}<p\right]=1$. So the desired inequalities hold at both endpoints of $[0,1]$, and we'd like to reason about $p \in(0,1)$. To accomplish this, it will actually be easier to compare $\operatorname{Pr}\left[Z_{(1), c}>p\right] \cdot \operatorname{Pr}\left[X_{(1), n}<p\right]$ to $\operatorname{Pr}\left[W_{\ell, n}>p \wedge X_{(1), n}<p\right]$ (observe that this simply multiplies both conditional probabilities in our original comparison by $\operatorname{Pr}\left[X_{(1), n}<p\right]$ ), and consider the derivative with respect to $p$.

So let $f_{1, n}(\cdot)$ denote the density of $X_{(1), n}$. Then $\operatorname{Pr}\left[W_{\ell, n}>\right.$ $\left.p \wedge X_{(1), n}<p\right]=\int_{0}^{p} f_{1, n}(q) \cdot \operatorname{Pr}\left[W_{\ell, n}>p \mid X_{(1), n}=q\right] d q$. By Leibniz' rule, the derivative of this with respect to $p$ is:

$$
\begin{aligned}
& \frac{\partial \operatorname{Pr}\left[W_{\ell, n}>p \wedge X_{(1), n}<p\right]}{\partial p} \\
= & \frac{\partial \int_{0}^{p} f_{1, n}(q) \cdot \operatorname{Pr}\left[W_{\ell, n}>p \mid X_{(1), n}=q\right] d q}{\partial p} \\
= & f_{1, n}(p) \cdot \operatorname{Pr}\left[W_{\ell, n}>p \mid X_{(1), n}=p\right] \\
+ & \int_{0}^{p} f_{1, n}(q) \cdot \frac{\partial \operatorname{Pr}\left[W_{\ell, n}>p \mid X_{(1), n}=q\right]}{\partial p} d q .
\end{aligned}
$$

Let's first unpack the left-most term with the following lemma.

Lemma 2. For all $\ell, n>1, \operatorname{Pr}\left[W_{\ell, n}>p \mid X_{(1), n}=p\right]=$ $\operatorname{Pr}\left[W_{\ell-1, n-1}>p \mid X_{(1), n-1}<p\right]$.

Proof. Observe that, conditioned on $X_{(1), n}=p, X_{(2), n}, \ldots$, $X_{(n), n}$ are $n-1$ (sorted) i.i.d. draws uniformly at random from $[0, p]$, and $X_{(\ell), n}$ is the $(\ell-1)^{t h}$ highest of them. Put another way, $X_{(\ell), n}$ conditioned on $X_{(1), n}=p$ is identically distributed to $X_{(\ell-1), n-1}$ conditioned on $X_{(1), n-1}<p$. This therefore implies that $W_{\ell, n}$ conditioned on $X_{(1), n}=p$ and $W_{\ell-1, n-1}$ conditioned on $X_{(1), n-1}<p$ are identically distributed as well.

Now we turn to the right-most term.

Lemma 3. For all $p, \ell, n, q$,

$\frac{\partial \operatorname{Pr}\left[W_{\ell, n}>p \mid X_{(1), n}=q\right]}{\partial p}=-\operatorname{Pr}\left[W_{\ell, n}>p \mid X_{(1), n}=q\right] /(1-p)$

Proof. Let's first expand $\operatorname{Pr}\left[W_{\ell, n}>p \mid X_{(1), n}=q\right]$ by letting $f_{\ell, n}^{q}(\cdot)$ denote the density of $X_{(\ell), n}$ conditioned on $X_{(1), n}=q$.

$$
\operatorname{Pr}\left[W_{\ell, n}>p \mid X_{(1), n}=q\right]=\int_{0}^{q} f_{\ell, n}^{q}(r) \cdot \frac{1-p}{1-r} d r .
$$

This is simply because, conditioned on $X_{(\ell), n}=r$, the probability that $W_{\ell, n}$ (a uniformly random draw from $[r, 1]$ ) exceeds $p$ is exactly $\frac{1-p}{1-r}$. Taking now the derivative with respect to $p$ (again by Leibniz' rule), we see that:

$$
\begin{aligned}
& \frac{\partial \operatorname{Pr}\left[W_{\ell, n}>p \mid X_{(1), n}=q\right]}{\partial p} \\
= & -\int_{0}^{q} f_{\ell, n}^{q}(r) /(1-r) d r \\
= & -\operatorname{Pr}\left[W_{\ell, n}>p \mid X_{(1), n}=q\right] /(1-p) .
\end{aligned}
$$

Using Lemma 3, we can now rewrite:

$$
\begin{aligned}
& \int_{0}^{p} f_{1, n}(q) \cdot \frac{\partial \operatorname{Pr}\left[W_{\ell, n}>p \mid X_{(1), n}=q\right]}{\partial p} d q \\
= & \frac{-1}{1-p} \int_{0}^{p} f_{1, n}(q) \cdot \operatorname{Pr}\left[W_{\ell, n}>p \mid X_{(1), n}=q\right] d q \\
= & \frac{-\operatorname{Pr}\left[W_{\ell, n}>p \wedge X_{(1), n}<p\right]}{1-p}
\end{aligned}
$$

And using both Lemmas 2 and 3 we can now simplify (the second equality follows by recalling that $f_{1, n}(\cdot)$ is the density of $\left.X_{(1), n}\right)$ :

$$
\begin{aligned}
& \frac{\partial \operatorname{Pr}\left[W_{\ell, n}>p \wedge X_{(1), n}<p\right]}{\partial p} \\
= & f_{1, n}(p) \cdot \operatorname{Pr}\left[W_{\ell-1, n-1}>p \mid X_{(1), n-1}<p\right] \\
- & \frac{\operatorname{Pr}\left[W_{\ell, n}>p \wedge X_{(1), n}<p\right]}{1-p} \\
= & n p^{n-1} \cdot \operatorname{Pr}\left[W_{\ell-1, n-1}>p \mid X_{(1), n-1}<p\right] \\
& -\frac{\operatorname{Pr}\left[W_{\ell, n}>p \wedge X_{(1), n}<p\right]}{1-p}
\end{aligned}
$$

From here we'll show that whenever $\operatorname{Pr}\left[W_{\ell, n}>p \wedge X_{(1), n}<\right.$ $p] \geq \operatorname{Pr}\left[Z_{(1), c}>p \wedge X_{(1), n}<p\right]$ (i.e. whenever what we're 
trying to prove at $p$ is violated), then the derivative trends towards satisfying our desired claim.

Lemma 4. If $\operatorname{Pr}\left[W_{\ell, n}>p \wedge X_{(1), n}<p\right] \geq \operatorname{Pr}\left[Z_{(1), c}>p \wedge\right.$ $\left.X_{(1), n}<p\right]$, then:

$\frac{\partial \operatorname{Pr}\left[Z_{(1), c}>p \wedge X_{(1), n}<p\right]-\operatorname{Pr}\left[W_{\ell, n}>p \wedge X_{(1), n}<p\right]}{\partial p} \geq 0$.

Proof. Observe first that $\operatorname{Pr}\left[Z_{(1), c}>p \wedge X_{(1), n}<p\right]=$ $p^{n} \cdot\left(1-p^{c}\right)$. As such, we also have $\frac{\partial \operatorname{Pr}\left[Z_{(1), c}>p \wedge X_{(1), n}<p\right]}{\partial p}=$ $n p^{n-1}\left(1-p^{c}\right)-c p^{n+c-1}$. So if the hypotheses of the lemma are satisfied, then by Equation (5) we can write:

$$
\begin{aligned}
& \frac{\partial \operatorname{Pr}\left[Z_{(1), c}>p \wedge X_{(1), n}<p\right]-\operatorname{Pr}\left[W_{\ell, n}>p \wedge X_{(1), n}<p\right]}{\partial p} \\
& =n p^{n-1}\left(1-p^{c}\right)-c p^{n+c-1} \\
& -\left(n p^{n-1} \cdot \operatorname{Pr}\left[W_{\ell-1, n-1}>p \mid X_{(1), n-1}<p\right]\right. \\
& \left.-\frac{\operatorname{Pr}\left[W_{\ell, n}>p \wedge X_{(1), n}<p\right]}{1-p}\right) \\
& \geq n p^{n-1}\left(1-p^{c}\right)-c p^{n+c-1} \\
& -n p^{n-1} \cdot\left(1-p^{4(n-1) /(\ell-2)}\right)+\frac{p^{n}\left(1-p^{c}\right)}{1-p} .
\end{aligned}
$$

In the inequality, we have used two facts. First, we have used the inductive hypothesis, which claims that $\operatorname{Pr}\left[W_{\ell-1, n-1}>p \mid X_{(1), n-1}<\right.$ $p] \leq \operatorname{Pr}\left[Z_{(1), 4(n-1) /(\ell-2)}>p \mid X_{(1), n-1}<p\right]=1-p^{4(n-1) /(\ell-2)}$. Second, we have used the hypothesis of the lemma statement. Next, we can substitute $\left(1-p^{c}\right)=\left(1+p+\ldots+p^{c-1}\right) \cdot(1-p)$ (and make some other algebraic simplifications) to get:

$$
\begin{aligned}
& \frac{\partial \operatorname{Pr}\left[Z_{(1), c}>p \wedge X_{(1), n}<p\right]-\operatorname{Pr}\left[W_{\ell, n}>p \wedge X_{(1), n}<p\right]}{\partial p} \\
& \geq-(n+c) p^{n+c-1}+n p^{n-1+4(n-1) /(\ell-2)}+p^{n} \cdot \sum_{j=0}^{c-1} p^{j} .
\end{aligned}
$$

Recall again that we are hoping to prove that the above term is $\geq 0$. As $p \geq 0$, the above term is $\geq 0$ if and only if (dividing all terms by $p^{n-1}$ and rearranging):

$$
n p^{4(n-1) /(\ell-2)}+\sum_{j=1}^{c} p^{j} \geq(n+c) p^{c} .
$$

To conclude that Equation (10) indeed holds for all $p \in[0,1]$, consider the convex function $f(x):=p^{x}$, and the random variable $A$ where $A=j$ with probability $1 /(n+c)$ for all $j \in\{1, \ldots, c\}$, and $A=4(n-1) /(\ell-2)$ with probability $n /(n+c)$. Then the lefthand side of the equation above is exactly $(n+c) \cdot \mathbb{E}[f(A)]$. Therefore, we may conclude by Jensen's inequality that the left-hand side exceeds $(n+c) \cdot f(\mathbb{E}[A])=(n+c) \cdot p^{\frac{4(n-1) n /(\ell-2)+c(c+1) / 2}{n+c}}$ As $p \in[0,1],(n+c) \cdot p^{\frac{4(n-1) n /(\ell-2)+c(c+1) / 2}{n+c}} \geq(n+c) p^{c}$ if and only if $\frac{4(n-1) n /(\ell-2)+c(c+1) / 2}{n+c} \leq c$. So finally, our only remaining job is to see what values of $c$ satisfy:

$$
\frac{4(n-1) n}{\ell-2}+\frac{c(c+1)}{2} \leq n c+c^{2} .
$$

Indeed, observe that when $c=4 n /(\ell-1)$, we get:

$$
\begin{gathered}
\frac{4(n-1) n}{\ell-2}+\frac{c(c+1)}{2} \stackrel{?}{\leq} n c+c^{2} \\
\frac{4}{\ell-2}-\frac{4}{n(\ell-2)}+\frac{4^{2}}{2(\ell-1)^{2}}+\frac{4}{2 n(\ell-1)} \\
\stackrel{?}{\leq} \frac{4}{\ell-1}+\frac{4^{2}}{(\ell-1)^{2}} \\
2(\ell-1)^{2}-2(\ell-1)^{2} / n+4(\ell-2)+(\ell-2)(\ell-1) / n \\
\quad \stackrel{?}{\leq} 2(\ell-2)(\ell-1)+2 \cdot 4 \cdot(\ell-2) \\
2(\ell-1)-\ell(\ell-1) / n \stackrel{?}{\leq} 4 \cdot(\ell-2) \\
2 \frac{\ell-1}{\ell-2}-\frac{\ell(\ell-1)}{(\ell-2) n} \stackrel{?}{\leq} 4
\end{gathered}
$$

The last inequality indeed holds as $\ell \geq 3$.

Now we are ready to wrap up the proof of the proposition. We have just shown (Lemma 4 ) that for all $p$, either $\operatorname{Pr}\left[Z_{(1), c}>\right.$ $\left.p \wedge X_{(1), n}<p\right]-\operatorname{Pr}\left[W_{\ell, n}>p \wedge X_{(1), n}<p\right] \geq 0$, or $\frac{\partial \operatorname{Pr}\left[Z_{(1), c}>p \wedge X_{(1), n}<p\right]}{\partial p}-\frac{\partial \operatorname{Pr}\left[W_{\ell, n}>p \wedge X_{(1), n}<p\right]}{\partial p} \geq 0$. We now are ready to claim that this implies that $\operatorname{Pr}\left[Z_{(1), c}>p \wedge X_{(1), n}<\right.$ $p]-\operatorname{Pr}\left[W_{\ell, n}>p \wedge X_{(1), n}<p\right] \geq 0$ for all $p \in[0,1]$.

Indeed, define $G(p):=\operatorname{Pr}\left[Z_{(1), c}>p \wedge X_{(1), n}<p\right]-\operatorname{Pr}\left[W_{\ell, n}>\right.$ $\left.p \wedge X_{(1), n}<p\right]$. Then we have shown that for all $p$, either $G(p) \geq 0$ or $G^{\prime}(p) \geq 0$. Moreover, we know that $G(0)=0-0=0$. So assume for contradiction that there exists some $p$ with $G(p)<0$. Then because $G(\cdot)$ is continuous (and $G(0)=0$ ), there exists some open interval $(q, p)$ such that $G(x)<0$ on $(q, p)$, while $G(q)=0$. But now we have a contradiction: By Lemma $4, G^{\prime}(x) \geq 0$ on $(q, p)$, and $G(q)=0$. Therefore, we must also have $G(x) \geq 0$ on $(q, p)$, contradicting our initial assumption.

Therefore, we cannot have $G(x)<0$ anywhere on [0,1], meaning that $\operatorname{Pr}\left[Z_{(1), c}>p \wedge X_{(1), n}<p\right]-\operatorname{Pr}\left[W_{\ell, n}>p \wedge X_{(1), n}<\right.$ $p] \geq 0$. This is identical to the claim that $\operatorname{Pr}\left[Z_{(1), c}>p \mid X_{(1), n}<\right.$ $p] \geq \operatorname{Pr}\left[W_{\ell, n}>p \mid X_{(1), n}<p\right]$. This completes the proof of the proposition.

Theorem 7 now follows nearly directly from Proposition 4.

Proof of Theorem 7. We can directly compute $\operatorname{Pr}\left[X_{S}(n, c)>p\right]=\operatorname{Pr}\left[X_{(1), n}>p\right]+\operatorname{Pr}\left[Z_{(1), c}>p \mid X_{(1), n}<\right.$ $p] \cdot \operatorname{Pr}\left[X_{(1), n}<p\right]$. Similarly, $\operatorname{Pr}\left[X_{B}(n, \ell)>p\right]=\operatorname{Pr}\left[X_{(1), n}>\right.$ $p]+\operatorname{Pr}\left[W_{\ell, n}>p \mid X_{(1), n}<p\right] \cdot \operatorname{Pr}\left[X_{(1), n}<p\right]$. By Proposition 4, when $c \geq 4 n /(\ell-1)$, we get:

$$
\operatorname{Pr}\left[Z_{(1), c}>p \mid X_{(1), n}<p\right] \geq \operatorname{Pr}\left[W_{\ell, n}>p \mid X_{(1), n}<p\right] .
$$

Therefore,

$$
\begin{aligned}
& {\left[X_{(1), n}>p\right]+\operatorname{Pr}\left[Z_{(1), c}>p \mid X_{(1), n}<p\right] \cdot \operatorname{Pr}\left[X_{(1), n}<p\right] } \\
\geq & \operatorname{Pr}\left[X_{(1), n}>p\right]+\operatorname{Pr}\left[W_{\ell, n}>p \mid X_{(1), n}<p\right] \cdot \operatorname{Pr}\left[X_{(1), n}<p\right] .
\end{aligned}
$$

This implies

$$
\operatorname{Pr}\left[X_{S}(n, c)>p\right] \geq \operatorname{Pr}\left[W_{\ell, n}>p\right] .
$$


As the above holds for all $p \in[0,1]$, this proves that $X_{S}(n, c)$ stochastically dominates $X_{B}(n, \ell)$.

Theorem 8 will require one more similar proposition.

Proposition 5. Let $c \geq n \cdot(1+\ln (1+m / n))$, then for all $p$, $\operatorname{Pr}\left[Z_{(1), c}>p \mid X_{(1), n}<p\right] \geq \operatorname{Pr}\left[Y_{n, m-1}^{*}>p \mid X_{(1), n}<p\right]$, where $Y_{n, m-1}^{*}$ is the random variable equal to 0 if $Y_{1, m-1}<X_{(1), n}$, and is otherwise equal to $Y_{j, m-1}$ for a uniformly random $j \in\left\{j \mid Y_{j, m-1}>\right.$ $\left.X_{(1), n}\right\}$.

Proof. The proof of Proposition 5 is more direct than that of Proposition 4. This time, we can just directly compute $\operatorname{Pr}\left[Y_{n, m-1}^{*}>\right.$ $\left.p \mid X_{(1), n}<p\right]$. We again begin by observing that $\operatorname{Pr}\left[Z_{(1), c}>\right.$ $\left.p \mid X_{(1), n}<p\right]=\operatorname{Pr}\left[Z_{(1), c}>p\right]=1-p^{c}$.

We now turn to $Y_{n, m-1}^{*}$. Observe first that $Y_{n, m-1}^{*}=0$, conditioned on $X_{(1), n}=q$, with probability exactly $q^{m-1}$. This is because $Y_{n, m-1}^{*}$ is 0 whenever each of $m-1$ i.i.d. draws uniformly from $[0,1]$ are all $<q$. Now, conditioned on $Y_{n, m-1}^{*}>0$ (and also $X_{(1), n}=q$ ), observe that $Y_{n, m-1}^{*}$ is just a random draw from the uniform distribution on $\left[X_{(1), n}, 1\right]$. This is because, conditioned on $Y_{n, m-1}^{*}>0$ and $X_{(1), n}=q, Y_{n, m-1}^{*}$ simply picks uniformly at random among some number of i.i.d. random variables drawn uniformly from $\left[X_{(1), n}, 1\right]$. Therefore, conditioned on $Y_{n, m-1}^{*}>0$, and $X_{(1), n}=q, Y_{n, m-1}^{*}$ exceeds $p$ with probability exactly $\frac{1-p}{1-q}$. Therefore, we can compute (below, let $f_{1}(\cdot)$ denote the density of $X_{(1), n}$, and $F_{1}(\cdot)$ denote the $\left.\mathrm{CDF}\right)$ :

$$
\begin{aligned}
& \operatorname{Pr}\left[Y_{n, m-1}^{*}>p \mid X_{(1), n}<p\right] \\
= & \int_{0}^{p} \frac{f_{1}(q)}{F_{1}(p)} \cdot \operatorname{Pr}\left[Y_{n, m-1}^{*}>0 \mid X_{(1), n}=q\right] \\
& \cdot \operatorname{Pr}\left[Y_{n, m-1}^{*}>p \mid Y_{n, m-1}^{*}>0 \wedge X_{(1), n}=q\right] d q \\
= & \int_{0}^{p} \frac{n q^{n-1}}{p^{n}} \cdot\left(1-q^{m-1}\right) \cdot \frac{1-p}{1-q} d q \\
= & \frac{n(1-p)}{p^{n}} \cdot \int_{0}^{p} q^{n-1} \cdot\left(\sum_{i=0}^{m-2} q^{i}\right) d q \\
= & \frac{n(1-p)}{p^{n}} \cdot\left[\sum_{i=0}^{m-2} q^{i+n} /(n+i)\right]_{0}^{p} \\
= & \frac{n(1-p)}{p^{n}} \cdot \sum_{i=0}^{m-2} p^{i+n} /(n+i) \\
= & n(1-p) \cdot \sum_{i=0}^{m-2} p^{i} /(n+i) \\
= & 1-n p^{m-1} /(n+m-2) \\
& +n \cdot \sum_{i=1}^{m-2} p^{i}(1 /(n+i)-1 /(n+i-1)) \\
= & 1-n p^{m-1} /(n+m-2)-\sum_{i=1}^{m-2} n p^{i} /(n+i)(n+i-1)
\end{aligned}
$$

Before proceeding, we quickly observe that the sums of the coefficients of non-zero powers of $p$ is -1 (that is, $n /(n+m-$ $\left.2)+\sum_{i=1}^{m-2} n /(n+i)(n+i-1)=1\right)$. This is because the thirdfrom-the-bottom equality is clearly equal to 0 when $p=1$, and so the bottom equality must be equal to 0 when $p=1$ as well.

$$
\begin{gathered}
\operatorname{Pr}\left[Z_{(1), c}>p \mid X_{(1), n}<p\right] \stackrel{?}{\geq} \operatorname{Pr}\left[Y_{n, m-1}^{*}>p \mid X_{(1), n}<p\right] \\
1-p^{c} \stackrel{?}{\geq} \\
1-n p^{m-1} /(n+m-2)-\sum_{i=1}^{m-2} n p^{i} /(n+i)(n+i-1) \\
p^{c} \stackrel{?}{\leq} n p^{m-1} /(n+m-2)+\sum_{i=1}^{m-2} n p^{i} /(n+i)(n+i-1)
\end{gathered}
$$

From here, we again apply Jensen's inequality. Let $f(x):=p^{x}$ (which is convex), and let $A$ denote the random variable which is equal to $m-1$ with probability $n /(n+m-2)$ and equal to $i$ with probability $n /(n+i)(n+i-1)$ for all $i \in\{1, \ldots, m-2\}$. By reasoning in the previous paragraph (that the coefficients of non-zero powers of $p$ sum to -1 ), this is indeed a distribution. Then Jensen's inequality asserts that $\mathbb{E}[f(A)] \geq f(\mathbb{E}[A])$. Moreover, the right-hand side above is exactly $\mathbb{E}[f(A)]$. As such, we get that:

$$
\begin{aligned}
& n p^{m-1} /(n+m-2)+\sum_{i=1}^{m-2} n p^{i} /(n+i)(n+i-1) \\
\geq & p^{n(m-1) /(n+m-2)+\sum_{i=1}^{m-2} n i /(n+i)(n+i-1)} .
\end{aligned}
$$

As $p \in[0,1]$, this means that our desired inequality is satisfied as long as $c \geq n(m-1) /(n+m-2)+\sum_{i=1}^{m-2} n i /(n+i)(n+i-1)$. But now observe that:

$$
\begin{aligned}
& n(m-1) /(n+m-2)+\sum_{i=1}^{m-2} n i /(n+i)(n+i-1) \\
= & n\left(\frac{m-1}{n+m-2}+\sum_{i=1}^{m-2} \frac{i}{(n+i)(n+i-1)}\right) \\
\leq & n \cdot\left(1+\sum_{i=1}^{m-2} 1 /(n+i)\right) \\
\leq & n \cdot\left(1+\ln \left(\frac{n+m-2}{n}\right)\right) \\
\leq & n \cdot\left(1+\ln \left(1+\frac{m}{n}\right)\right) .
\end{aligned}
$$

And now we can prove Theorem 8, which essentially combines Proposition 4 and Proposition 5 (with some extra work).

Proof of Theorem 8. We again directly compute $\operatorname{Pr}\left[X_{S}(n, c)>p\right]=\operatorname{Pr}\left[X_{(1), n}>p\right]+\operatorname{Pr}\left[Z_{(1), c}>p \mid X_{(1), n}<p\right]$. Similarly, $\operatorname{Pr}\left[X_{L}(n, m)>p\right]=\operatorname{Pr}\left[X_{(1), n}>p\right]+\operatorname{Pr}\left[W_{2, n}>\right.$ $\left.p \mid X_{(1), n}<p\right] \cdot \operatorname{Pr}\left[X_{(1), n}<p\right]+\operatorname{Pr}\left[Y_{n, m-1}^{*}>p \mid X_{(1), n}<\right.$ $\left.p \wedge W_{2, n}<p\right] \cdot \operatorname{Pr}\left[X_{(1), n}<p \wedge W_{2, n}<p\right]$, where again $Y_{n, m-1}^{*}$ is defined to be 0 if $Y_{1, m-1}<X_{(1), n}$, and otherwise equal to $Y_{j, m-1}$ for a uniformly random $j \in\left\{j \mid Y_{j, m-1}>X_{(1), n}\right\}$.

By Proposition 4 we have that $\operatorname{Pr}\left[W_{2, n}>p \mid X_{(1), n}<p\right] \leq$ $\operatorname{Pr}\left[Z_{(1), n}>p\right]$. Now we need to reason about $Y_{n, m-1}^{*}$ conditioned on $X_{(1), n}<p$ and $W_{2, n}<p$, which is not directly related to any previous propositions. However, we claim that $Y_{n, m-1}^{*}$ and $W_{2, n}$ are positively correlated, conditioned on $X_{(1), n}<p$. 
Lemma 5. $\operatorname{Pr}\left[Y_{n, m-1}^{*}>p \mid X_{(1), n}<p \wedge W_{2, n}<p\right] \leq$ $\operatorname{Pr}\left[Y_{n, m-1}^{*}>p \mid X_{(1), n}<p\right]$.

Proof. For this proof, for random variables $A, B, C$, when we say $A$ and $B$ are conditionally independent, conditioned on $C$ we mean that for all $c$, the random variables $A$ and $B$ are conditionally independent, conditioned on the event $C=c$. We use this shorthand to avoid cumbersome notation.

The proof consists of three steps: (a) we first show $Y_{n, m-1}^{*}$ and $W_{2, n}$ are conditionally independent, conditioned on $X_{(1), n}$, (b) we show that, conditioned on $X_{(1), n}<p, Y_{n, m-1}^{*}$ is positively correlated with $X_{(1), n}$, and (c), $W_{2, n}$ is positively correlated with $X_{(1), n}$. Together, this essentially lets us claim that additionally conditioning on $W_{2, n}<p$ only serves to lower $X_{(1), n}$, which lowers the probability that $Y_{n, m-1}^{*}>p$.

Observe first that conditioned on $X_{(1), n}, \ldots, X_{(n), n}, Y_{n, m-1}^{*}$ and $W_{2, n}$ are independent (this is just by definition: they are drawn independently, but the distributions from which they are drawn depend on $\left.X_{(1), n}, \ldots, X_{(n), n}\right)$. However, observe that the distribution from which $Y_{n, m-1}^{*}$ is independently drawn can be defined as a function only of $X_{(1), n}$. This means that, conditioned on $X_{(1), n}$, $X_{(2), n}$ and $Y_{n, m-1}^{*}$ are conditionally independent. Similarly, the distribution from which $W_{2, n}$ is independently drawn from can be described as a function only of $X_{(2), n}$, which we just claimed is conditionally independent of $Y_{n, m-1}^{*}$, conditioned on $X_{(1), n}$. Therefore, $Y_{n, m-1}^{*}$ and $W_{2, n}$ are conditionally independent, conditioned on $X_{(1), n} \cdot{ }^{8}$

Next, we want to claim that, for all $r \leq q \leq p, \operatorname{Pr}\left[Y_{n, m-1}^{*}>\right.$ $\left.p \mid X_{(1), n}=q\right] \geq \operatorname{Pr}\left[Y_{n, m-1}^{*}>p \mid X_{(1), n}=r\right]$. To see this, observe the following equivalent method for drawing $Y_{n, m-1}^{*}$ : First, draw $Y_{1, m-1}, \ldots, Y_{m-1, m-1}$ i.i.d. from the uniform distribution on $[0,1]$. Permute them into random order. ${ }^{9}$ Then, let $j$ be the smallest index such that $Y_{j, m-1}>X_{(1), n}$. If no such $j$ exists, set $Y_{n, m-1}^{*}=0$. Otherwise, set $Y_{n, m-1}^{*}=Y_{j, m-1}$. Now, let's couple draws for $Y_{n, m-1}^{*}$ conditioned on $X_{(1), n}=q$ and $X_{(1), n}=r$ by fixing the values $Y_{1, m-1}, \ldots, Y_{m-1, m-1}$ and the random permutation. Then think of $Y_{n, m-1}^{*}$, conditioned on $X_{(1), n}=r$ (respectively, $X_{(1), n}=q$ ) as scanning the values sequentially until it hits one whose value exceeds $r$ (respectively, $q$ ).

- If the (permuted) sequence $Y_{1, m-1}, \ldots, Y_{m-1, m-1}$ has no values $>p$, we have $Y_{n, m-1}^{*}<p$ in both cases.

- If the sequence has values $>p$, but a value $\in(q, p)$ precedes all values $>p$, then again $Y_{n, m-1}^{*}<p$ in both cases. This is because both scans stop at the value $\in(q, p)$ which is not $>p$.

- If the sequence has value $>p$, and the first one is not preceded by any value $\in(r, p)$, then $Y_{n, m-1}^{*}>p$ in both cases. This is because both scans stop at a value $>p$ and output it.

- If the sequence has a value $>p$, but a value $\in(r, q)$ precedes all values $>p$ but no value $\in(q, p)$ precedes the first value $>p$ : then $Y_{n, m-1}^{*}>p$ when conditioned on $X_{(1), n}=q$, but $Y_{n, m-1}^{*}<p$ when conditioned on $X_{(1), n}=r$. This is because the $X_{(1), n}=q$ scan skips over the value $\in(r, q)$,

${ }^{8}$ Note that $Y_{n, m-1}^{*}$ and $W_{2, n}$ are not conditionally independent, conditioned on $X_{(1), n}<p$. They are only conditionally independent, conditioned on $X_{(1), n}=q$ (for some $q$ ).

${ }^{9}$ Actually, this step is not necessary, but it helps the analogy to state it. and stops at the value $>p$, whereas the $X_{(1), n}=r$ scan stops at the value $\in(r, q)$.

This covers all cases, and proves that for all $r \leq q \leq p$, $\operatorname{Pr}\left[Y_{n, m-1}^{*}>p \mid X_{(1), n}=q\right] \geq \operatorname{Pr}\left[Y_{n, m-1}^{*}>p \mid X_{(1), n}=r\right]$.

Finally, we make the same claim for $W_{2, \ell}$ : for all $r \leq q, \operatorname{Pr}\left[W_{2, \ell}>\right.$ $\left.p \mid X_{(1), n}=q\right] \geq \operatorname{Pr}\left[W_{2, \ell}>p \mid X_{(1), n}=r\right]$. This claim is more straight-forward: $W_{2, \ell}$ is drawn from a uniform distribution on $\left[X_{(2), \ell}, 1\right]$. So clearly, $\operatorname{Pr}\left[W_{2, \ell}>p \mid X_{(2), n}=q\right] \geq \operatorname{Pr}\left[W_{2, \ell}>\right.$ $\left.p \mid X_{(2), n}=r\right]$ whenever $r \leq q$. Moreover, $X_{(2), n}$ is distributed according to the maximum of $n-1$ i.i.d. uniform draws from $\left[0, X_{(1), n}\right]$, so the distribution of $X_{(2), n}$ conditioned on $X_{(1), n}=q$ stochastically dominates that of $X_{(2), n}$ conditioned on $X_{(1), n}=r$ whenever $q \geq r$. Both observations together allow us to conclude that for all $r \leq q, \operatorname{Pr}\left[W_{2, \ell}>p \mid X_{(1), n}=q\right] \geq \operatorname{Pr}\left[W_{2, \ell}>\right.$ $\left.p \mid X_{(1), n}=r\right]$.

Now we may put all three claims together to prove the lemma.

Now with Lemma 5, we can wrap up the proof. We now know that:

$$
\begin{aligned}
& \operatorname{Pr}\left[X_{L}(n, m)>p\right] \\
= & \operatorname{Pr}\left[X_{(1), n}>p\right]+\operatorname{Pr}\left[W_{2, n}>p \mid X_{(1), n}<p\right] \cdot \operatorname{Pr}\left[X_{(1), n}<p\right] \\
& +\operatorname{Pr}\left[Y_{n, m-1}^{*}>p \mid X_{(1), n}<p \wedge W_{2, n}<p\right] \\
& +\operatorname{Pr}\left[X_{(1), n}<p \wedge W_{2, n}<p\right] \\
\leq & \operatorname{Pr}\left[X_{(1), n}>p\right] \\
& +\operatorname{Pr}\left[W_{2, n}>p \mid X_{(1), n}<p\right] \cdot \operatorname{Pr}\left[X_{(1), n}<p\right] \\
& +\operatorname{Pr}\left[Y_{n, m-1}^{*}>p \mid X_{(1), n}<p\right] \cdot \operatorname{Pr}\left[X_{(1), n}<p \wedge W_{2, n}<p\right] \\
\leq & \operatorname{Pr}\left[X_{(1), n}>p\right] \\
& +\operatorname{Pr}\left[W_{2, n}>p \mid X_{(1), n}<p\right] \cdot \operatorname{Pr}\left[X_{(1), n}<p\right] \\
& +\operatorname{Pr}\left[Z_{(1), n(1+\ln (1+m / n))}>p \mid X_{(1), n}<p\right] \cdot \\
& \operatorname{Pr}\left[X_{(1), n}<p \wedge W_{2, n}<p\right] \\
\leq & \operatorname{Pr}\left[X_{(1), n}>p\right] \\
& +\operatorname{Pr}\left[W_{2, n}>p \vee Z_{(1), n(1+\ln (1+m / n))}>p \mid X_{(1), n}<p\right] \\
& +\operatorname{Pr}\left[X_{(1), n}<p\right] \\
& +
\end{aligned}
$$

At this point, observe that the random variables $W_{2, n}$ and $Z_{(1), n(1+\ln (1+m / n))}$ are independent (and also conditionally independent, conditioned on $\left.X_{(1), n}<p\right)$. Therefore:

$$
\begin{aligned}
& \operatorname{Pr}\left[W_{2, n}<p \wedge Z_{(1), n(1+\ln (1+m / n))}<p \mid X_{(1), n}<p\right] \\
= & \operatorname{Pr}\left[W_{2, n}<p \mid X_{(1), n}<p\right] \\
& \cdot \operatorname{Pr}\left[Z_{(1), n(1+\ln (1+m / n))}<p \mid X_{(1), n}<p\right]
\end{aligned}
$$

By Proposition 4, we know that $\operatorname{Pr}\left[W_{2, n}<p \mid X_{(1), n}<p\right] \geq$ $\operatorname{Pr}\left[Z_{(1), n}<p \mid X_{(1), n}<p\right]$. Therefore, we get that:

$$
\begin{aligned}
& \operatorname{Pr}\left[W_{2, n}<p \wedge Z_{(1), n(1+\ln (1+m / n))}<p \mid X_{(1), n}<p\right] \\
\geq & \operatorname{Pr}\left[Z_{(1), n}<p \mid X_{(1), n}<p\right] \\
& \cdot \operatorname{Pr}\left[Z_{(1), n(1+\ln (1+m / n))}<p \mid X_{(1), n}<p\right] \\
= & \operatorname{Pr}\left[Z_{(1), n}<p\right] \cdot \operatorname{Pr}\left[Z_{(1), n(1+\ln (1+m / n))}<p\right] \\
= & \operatorname{Pr}\left[Z_{(1), n(2+\ln (1+m / n))}<p\right] .
\end{aligned}
$$


Therefore,

$$
\begin{aligned}
& \operatorname{Pr}\left[Z_{(1), n(2+\ln (1+m / n))}>p\right] \\
\geq & \operatorname{Pr}\left[W_{2, n}>p \vee Z_{(1), n(1+\ln (1+m / n))}>p \mid X_{(1), n}<p\right] .
\end{aligned}
$$

So substituting all the way back, we get that:

$$
\begin{aligned}
& {\left[X_{L}(n, m)>p\right] } \\
\leq & \operatorname{Pr}\left[X_{(1), n}>p\right]+\operatorname{Pr}\left[Z_{(1), n(2+\ln (1+m / n))}>p\right] \\
& \cdot \operatorname{Pr}\left[X_{(1), n}<p\right] \\
= & \operatorname{Pr}\left[X_{S}(n, n(2+\ln (1+m / n)))>p\right] .
\end{aligned}
$$

This completes the proof.

\section{REFERENCES}

[BCKW15] Patrick Briest, Shuchi Chawla, Robert Kleinberg, and S. Matthew Weinberg. Pricing lotteries. F. Economic Theory, 156:144-174, 2015.

[BGN17] Moshe Babaioff, Yannai A. Gonczarowski, and Noam Nisan. The menusize complexity of revenue approximation. In Proceedings of the 49th Annual ACM SIGACT Symposium on Theory of Computing, STOC 2017, Montreal, QC, Canada, fune 19-23, 2017, pages 869-877, 2017.

[BILW14] Moshe Babaioff, Nicole Immorlica, Brendan Lucier, and S. Matthew Weinberg. A simple and approximately optimal mechanism for an additive buyer. In the 55th Annual IEEE Symposium on Foundations of Computer Science (FOCS), 2014.

[BK96] Jeremy Bulow and Paul Klemperer. Auctions versus negotiations. The American Economic Review, pages 180-194, 1996.

[CDW16] Yang Cai, Nikhil Devanur, and S. Matthew Weinberg. A duality based unified approach to bayesian mechanism design. In Proceedings of the 48th ACM Conference on Theory of Computation(STOC), 2016. https://arxiv org/abs/1812.01577.

[CHK07] Shuchi Chawla, Jason D. Hartline, and Robert D. Kleinberg. Algorithmic Pricing via Virtual Valuations. In the 8th ACM Conference on Electronic Commerce (EC), 2007.

[CHMS10] Shuchi Chawla, Jason D. Hartline, David L. Malec, and Balasubramanian Sivan. Multi-Parameter Mechanism Design and Sequential Posted Pricing. In the 42nd ACM Symposium on Theory of Computing (STOC), 2010.

[CM16] Shuchi Chawla and J. Benjamin Miller. Mechanism design for subadditive agents via an ex ante relaxation. In Proceedings of the 2016 ACM Conference on Economics and Computation, EC '16, Maastricht, The Netherlands, fuly 24-28, 2016, pages 579-596, 2016.

[CMS15] Shuchi Chawla, David L. Malec, and Balasubramanian Sivan. The power of randomness in bayesian optimal mechanism design. Games and Economic Behavior, 91:297-317, 2015.

[CZ17] Yang Cai and Mingfei Zhao. Simple mechanisms for subadditive buyers via duality. In Proceedings of the 49th Annual ACM SIGACT Symposium on
Theory of Computing, STOC 2017, Montreal, OC, Canada, fune 19-23, 2017, pages $170-183,2017$.

[DDT17] Constantinos Daskalakis, Alan Deckelbaum, and Christos Tzamos. Strong duality for a multiple-good monopolist. Econometrica, 85(3):735-767, 2017.

$\left[\mathrm{EFF}^{+} 17 \mathrm{a}\right]$ Alon Eden, Michal Feldman, Ophir Friedler, Inbal Talgam-Cohen, and S. Matthew Weinberg. The competition complexity of auctions: A bulowklemperer result for multi-dimensional bidders. In Proceedings of the 2017 ACM Conference on Economics and Computation, EC '17, Cambridge, MA, USA, fune 26-30, 2017, page 343, 2017.

$\left[\mathrm{EFF}^{+}\right.$17b] Alon Eden, Michal Feldman, Ophir Friedler, Inbal Talgam-Cohen, and S. Matthew Weinberg. A simple and approximately optimal mechanism for a buyer with complements: Abstract. In Proceedings of the 2017 ACM Conference on Economics and Computation, EC '17, Cambridge, MA, USA, June 26-30, 2017, page 323, 2017.

[FFR18] Michal Feldman, Ophir Friedler, and Aviad Rubinstein. 99\% revenue via enhanced competition. In Proceedings of the 2018 ACM Conference on Economics and Computation, Ithaca, NY, USA, fune 18-22, 2018, pages 443-460, 2018.

[FLLT18] Hu Fu, Christopher Liaw, Pinyan Lu, and Zhihao Gavin Tang. The value of information concealment. In Proceedings of the Twenty-Ninth Annual ACM-SIAM Symposium on Discrete Algorithms, SODA 2018, New Orleans, LA, USA, fanuary 7-10, 2018, pages 2533-2544, 2018.

[Har11] Jason D. Hartline. Approximation and Mechanism Design. 2011.

[HN13] Sergiu Hart and Noam Nisan. The menu-size complexity of auctions. In the 14th ACM Conference on Electronic Commerce (EC), 2013

[HN17] Sergiu Hart and Noam Nisan. Approximate revenue maximization with multiple items. F. Economic Theory, 172:313-347, 2017.

[HR15] Sergiu Hart and Philip J. Reny. Maximizing Revenue with Multiple Goods: Nonmonotonicity and Other Observations. Theoretical Economics, 10(3):893-922, 2015.

[LP18] Siqi Liu and Christos-Alexandros Psomas. On the competition complexity of dynamic mechanism design. In Proceedings of the Twenty-Ninth Annual ACM-SIAM Symposium on Discrete Algorithms, SODA 2018, New Orleans, LA, USA, fanuary 7-10, 2018, pages 2008-2025, 2018.

[LY13] Xinye Li and Andrew Chi-Chih Yao. On revenue maximization for selling multiple independently distributed items. Proceedings of the National Academy of Sciences, 110(28):11232-11237, 2013.

[Mye81] Roger B. Myerson. Optimal Auction Design. Mathematics of Operations Research, 6(1):58-73, 1981.

[Pav11] Gregory Pavlov. Optimal mechanism for selling two goods. The B.E. Journal of Theoretical Economics, 11(3), 2011.

[RTCY12] Tim Roughgarden, Inbal Talgam-Cohen, and Qiqi Yan. Supply-limiting mechanisms. In 13th ACM Conference on Electronic Commerce (EC), 2012.

[RW15] Aviad Rubinstein and S. Matthew Weinberg. Simple mechanisms for a subadditive buyer and applications to revenue monotonicity. In Proceedings of the 16th ACM Conference on Electronic Commerce, 2015.

[Tha04] John Thanassoulis. Haggling over substitutes. fournal of Economic Theory, 117:217-245, 2004

[Yao15] Andrew Chi-Chih Yao. An n-to-1 bidder reduction for multi-item auctions and its applications. In the Twenty-Sixth Annual ACM-SIAM Symposium on Discrete Algorithms (SODA), 2015 